\title{
Analysis of the Incidence of Dental Pathologies in Ornamental and Agricultural Rabbits
}

\author{
Marina Kharitonova, Oksana Bokhina, and Sergey Klyukin* \\ Federal State-Owned Publicly-Funded Institution of Higher Education Saratov State Agrarian \\ University named after N.I. Vavilov, Saratov, 410012, Russia, 1 Teatralnaya pl.
}

\begin{abstract}
Studies of the incidence of dental diseases and pathologies in decorative and agricultural breeds of rabbits have been carried out, the most effective treatment and preventive measures have been substantiated. The article presents the results of an analysis with the statistics of the incidence of such dental pathologies as overgrowth of incisors, overgrowth of cheek teeth and uneven wear due to tooth loss, among all recorded diseases in rabbits of different age groups. The authors identified dental pathologies that occupy a significant share among all registered rabbit diseases in the Saratov region. Dental diseases appear in older animals, with the exception of the overgrowth of incisors, this pathology is often found in young individuals of decorative rabbits. Treatment of overgrowth of cheek teeth is presented in the elimination of sharp ends of teeth - hooks and thorns. Individual teeth are removed if their roots are infected and cause abscesses. Correction of cheek teeth is carried out most often for decorative rabbits for apartment maintenance. Less often, it is performed for high-yield agricultural rabbits, producers, if their pathology was not caused by a genetic predisposition, but was the result of injury or improper feeding. Simple filing of the incisors is carried out without sedation, using a secure fixation of the rabbit. If the incisors grow too quickly in the rabbit, they are extracted. The main method of preventive measures and recommendations for prevention of dental disease in a rabbit is to maintain an appropriate diet. For early detection and treatment of dental problems in rabbits, regular check-ups and dental evaluations are essential.
\end{abstract}

\section{Introduction}

The domestic rabbit has about 305 registered breeds, is a domesticated variety of the European rabbit ( Oryctolagus cuniculus L., 1758) and belongs to the order of Lagomorpha. Previously, representatives of this order were classified as rodents, but due to the peculiarities of anatomy and, in particular, the structure of the jaw apparatus, which is different from rodents, they were taken to a separate order [1]. In the practice of veterinary specialists, the number of cases of registration of detailed diseases of rabbits, which are noted with frequent relapses, has significantly increased. Diseases lead to decreased productivity in animals and are often fatal. Rabbits with dental diseases are rejected and are

\footnotetext{
*Corresponding author: klyukin15@mail.ru
} 
not intended for further breeding [2]. The most common cause of these diseases is the inheritance of an abnormal configuration of the bones of the skull and teeth. In recent years, due to the low level of breeding work in our country, the number of such animals has increased several times [3]. As a rule, hereditary diseases first appear already in the first year of life.

The study of the causes of dental diseases in rabbits, the use of optimal treatment methods, and most importantly, prevention is relevant and will lead to reduction in sick animals, an increase in the quality of products produced by rabbit farms and an improvement in the health of decorative rabbits [4].

Dental diseases of rabbits are caused by improper grinding of teeth, errors in feeding and zoohygiene, as well as genetic predisposition. Awareness of this problem during examination and clinical examination of rabbits will help a veterinarian to correctly diagnose these pathologies, provide veterinary care and exclude from breeding individuals with a tendency to these pathologies [5].

The most common dental problems in rabbits are overgrowth of cheek teeth and incisors, leading to malocclusion and disocclusion, abscesses, pathology of the nasolacrimal duct (dacryocystitis) and stomatitis [6-9]. Such diseases lead to the following pathological conditions:

- violation of the growth of the roots of the teeth;

- damage to the oral mucosa;

- formation of ulcers in the mouth and tongue;

- infection of the nasolacrimal canal;

- infection of the postorbital space;

- infection of bone tissue;

- development of purulent conjunctivitis;

- formation of postorbital abscesses;

- formation of abscesses of the lower jaw;

- osteomyelitis;

- disruption of the temporomandibular joint;

- exhaustion and dehydration of the animal due to refusal of food,

- development of tympania and/or atony of the gastrointestinal tract [4, 6-8].

The aim of the paper is to analyze the incidence of dental diseases in different breeds of rabbits and to substantiate the most effective therapeutic and prophylactic measures.

The dentition of rabbits is heterodont and consists of 28 teeth. In the upper jaw there are two incisors (I) - dens incisivusmajor and dens incisivus minor and six cheek teeth (three premolars (P) and three molars (M)). The mandible has one incisor and five cheek teeth (two premolars and three molars) that are present on both sides. Canine teeth are missing. There is a large diastema between incisors and premolars [9-11].

Premolars and molars are morphologically similar and form a series of chewing cheek teeth. The mandibular cheek teeth are in a straight line. The maxillary cheek teeth are approximately the same, except for the first premolar and the last molar, which are narrower than the middle teeth, which gives the cheek edge a curved shape. The shape of the teeth is constantly changing. They tend to bend at first and then bend progressively. The upper teeth are laterally, the lower teeth are towards the tongue.

The cheek surfaces of the rubbing teeth are anisognathic, that is, the lower teeth are thinner than the upper ones. When the jaws are closed, the lower cheek teeth are inside the upper ones, and the cheek edge of the lower teeth only slightly touches the palatal edge of the upper ones [10-13]. When chewing food, this structure of the jaws allows to make the process itself more efficient.

The incisors are used to cut through vegetation like scissors. The lower incisors move laterally to grind coarse food. The large upper primary incisors have a thick layer of enamel 
on the outside. This distribution of enamel allows the formation of sharp cutting edges at the ends of the upper primary incisors. During chewing, the jaw moves in an arc that also brings the incisors of the lower jaw into contact with the teeth of the upper jaw. This action leads to wear of the tips of the incisors of the lower jaw to a sharp edge.

In healthy rabbits, the process of sharpening teeth occurs due to their constant growth and wear. Rabbit incisors grow at an average rate of 10-37 microns per hour or 3001000 microns per day. Abrasion - occlusal wear of the upper and lower teeth, abutting each other. As a result, soft dentin wears off faster than hard enamel. Sharpening of teeth also occurs due to contact with food, teeth with each other and any other abrasive material.

Rabbit teeth have the same structural components as other animals, i.e. dentin, enamel, cementum and pulp. The main body of a tooth is dentin, which is made up of hydroxyapatite crystals similar to those found in bone, but much denser. The crystals are embedded in a collagen matrix, which is also similar to bone, but without osteocytes, osteoclasts, osteoblasts, or blood vessels. Dentin consists of a layer of odontoblasts lining its inner surface along the pulp cavity. This layer contains free sensory nerve endings, some of which pass into the dentin through the tubules. Species with constantly erupting teeth, such as rabbits, have fewer dentin axons than animals with permanent teeth [10]. The enamel is formed by a layer of ameloblasts [9]. The circumference of the tooth consists of enamel, covered with a layer of acellular cement, which encloses the fibers of the periodontal ligament. In the center of molars and premolars there is a deep longitudinal enamel fold.

The opposite ends of the fibers are embedded in the alveolar bone, fixing the tooth in the alveolar socket. Like incisors, cheek teeth maintain their shape through a continuous process of growth and wear. The softer cement and harder dentin of the occlusal surface wears away earlier than the enamel, which remains a sharp edge both around the circumference and in the center of the tooth. This gives molars and premolars an effective grinding surface. The enamel crease forms a ridge in the center of the occlusal surface that engages the interdental space between the two occlusal teeth [12].

The pathogenesis of dental diseases in rabbits can include a number of primary and secondary conditions such as inheritance related to the size and shape of the skull, trauma, metabolic deficiency or nutritional imbalance; however, a significant proportion of dental disease in rabbits is associated with insufficient tooth wear.

Normally, tooth growth occurs at the same time as teeth wear, so when crowns are not worn enough, elongated teeth put pressure on opposite teeth, creating occlusal stress that can slow tooth growth. This process can delay the eruption of tooth tissue, but cannot stop it completely, and delayed eruption can have detrimental consequences for development of tooth tissue, including deformation of new tooth tissue, formation of ridges on the enamel and, in severe cases, abnormal curvature and intrusive growth [14 ]. Additional problems can be associated with the growth of the root of the upper and lower jaw, for example, they can cause blockage of the nasolacrimal duct, put pressure on the orbit of the eye, and lead to the exit of the root through the ventral border of the lower jaw.

One of the most common dental problems encountered in rabbits is malocclusion, where the teeth do not meet normally. Almost without exception, malocclusion is accompanied by crown lengthening, as a result of which the growth rate exceeds the wear of the opposite occlusal surface [7-9]. This can be a serious problem for the rabbit, as its teeth can grow to abnormal length and shape, which interferes with effective chewing and increases the likelihood of health problems as the rabbit becomes increasingly unable to rub its teeth evenly.

Malocclusion can be seen in young animals if it is due to congenital or hereditary causes such as deformity of the length of the jaw. The curvature of the incisors leads to the fact that they grow into the oral cavity or, in some cases, overgrown teeth can bend and pierce 
the palate, which leads to damage to the soft tissues and protrusion into the anterior nasal cavity. An improper bite on the incisor can also prevent the rabbit from closing its mouth, leading to overgrowth of cheek teeth.

Malocclusion can also occur at any age as a result of trauma, malnutrition, infection or tooth loss, which subsequently leads to a change in the occlusal surface and can lead to a lack of resistance to occlusal forces and subsequent growth or deformation of the teeth. However, the most common cause of malocclusion is crown elongation due to insufficient wear during normal chewing activity, and this is usually seen in adult animals.

An incorrect bite can only be on the incisors, but more often affects the entire oral cavity. If the incisors do not join properly, it can lead to ineffective chewing and uneven wear on the cheek teeth. Reducing lateral movement during chewing can cause the formation of sharp enamel dots or spurs; natural anisognathism of the rabbit causes lingual spurs to form on the cheek teeth of the lower jaw, while cheek spurs form on the teeth of the upper jaw.

Spurs on the cheek teeth can severely damage the lining of mouth, leading to pain and infection. The rabbit may be reluctant to swallow, which can lead to hypersalvation. This is usually accompanied by anorexia, which can quickly lead to catastrophic gastrointestinal congestion.

Infections, followed by abscess and osteomyelitis, can occur in rabbits for a number of reasons. Occlusal stress due to misalignment of teeth can play a role in putting pressure on the roots of the teeth and leading to deformation of the teeth, thereby increasing the susceptibility to periodontal disease. In addition, crown lengthening can lead to an increase in the gap between teeth, a relatively weak periodontal ligament can lead to deeper periodontal pockets, which can be invaded by pathogenic bacteria.

Submandibular and retrobulbar abscesses are frequent companions of dental diseases, since the oral mucosa is damaged by sharp spurs on the teeth or displaced incisors, which led to development of periapical infection. This set of conditions, characterized by periodontal disease or endodontosis accompanied by submandibular or retrobulbar abscesses, is thought to be due in part to elevated parathyroid hormone levels accompanied by vitamin $\mathrm{D}$ and calcium deficiencies $[7,13,15]$.

Rabbit dental caries are points of decay and demineralization on a tooth caused by a bacterial infection. The acid produced by pathogenic bacteria destroys enamel and dentin, causing destruction of tooth tissue and leading to formation of holes in the surface of the tooth. This process can lead to pain, root infection, and tooth loss. This is a rare occurrence in rabbits due to the constant growth and replacement of dental tissue. However, with a diet high in carbohydrates, the disease appears in rabbits as well [14].

\section{Methods}

The objects of the study were decorative and agricultural rabbits of various breeds and ages with dental pathologies. A total of 618 rabbits were examined during the study period, 235 of which were decorative, apartment-based, 383 were agricultural ones, kept in cages, sheds and aviaries. In the Saratov region, rabbit breeding is developed in the conditions of private and peasant farms, where large and medium-sized meat (New Zealand White, Californian) and meat-skin breeds (Soviet chinchilla) are bred. In the conditions of apartment housing, decorative small or medium, downy (Angora, Lionhead) or skin-meat breeds (Russian ermine, Rex, Dwarf Rex, Alaska, Havanna) are often chosen as animal companions.

To determine the state of rabbit breeding in the Saratov region, as well as to identify the proportion of dental pathologies among other rabbit diseases, a dental examination of decorative and agricultural rabbits was carried out. It was determined that the 
brachycephalic breeds of rabbits are most often susceptible to dental pathologies, which is caused by a genetic predisposition as a result of selection.

\section{Results}

Among the decorative rabbits kept in apartments, the most common dental pathologies are found in the following breeds: Sheep (34\%), Dwarf Rex (12\%), Angora (8\%), Lionhead $(6 \%)$, Rex $(6 \%)$. In agricultural rabbits, the following breeds are more susceptible to dental diseases: California (28 \%), Black brown rabbit (22\%), New Zealand white (17\%).

The diagnosis of dental patients is made using standard and special study methods and includes taking anamnesis, a complete physical examination of the animal, examination of the oral cavity under sedation (using an otoscope), and x-rays.

Most often, a veterinarian is treated with animals that have had dental pathologies for a long time, and, therefore, have various complications of the underlying disease (exhaustion, liver lipidosis). Such patients are shown additional diagnostics - blood tests, ultrasound examination.

As a special study, we used X-ray of the dentoalveolar apparatus of rabbits. Standard Xray examination was performed under gas anesthesia, with at least five projections being performed: right lateral, dorsoventral or ventrodorsal, rostrocaudal, and two oblique ones. Intraoral images were taken if necessary $[4,5,14,16]$.

The dental examination was carried out according to the methods of the standard protocol for the examination of dental patients - rodents and rabbits. X-ray, ultrasound, general and biochemical blood tests were used as the additional special research methods. The teeth were corrected under sedation, gas anesthesia using the drug isoflurane. Antibiotics were also used - marbofloxacin $-5 \mathrm{mg} / \mathrm{kg} 1 \mathrm{t} / \mathrm{d}$. The course is from 5 days to several months, bitillin $3-30,000 \mathrm{U} / \mathrm{kg} 1 \mathrm{t} / 72 \mathrm{~h}$ and analgesics - meloxicam 0.1-0.2 mg/kg $1 \mathrm{t} / \mathrm{d}$, loxicom $0.2-0.4 \mathrm{ml} / \mathrm{kg} 1 \mathrm{t} / \mathrm{d}[7,17]$.

In the course of the studies carried out, we can say that dental pathologies are one of the most common problems that a veterinarian encounters when working with rabbits, both decorative and agricultural.

Table 1 shows the results of the analysis with the statistics of the occurrence of such dental pathologies as the overgrowth of incisors, overgrowth of cheek teeth and uneven wear due to tooth loss, among all recorded diseases in decorative and agricultural rabbits of different age groups.

Table 1. The incidence of various dental pathologies among other pathologies in young and old individuals of decorative and agricultural rabbits.

\begin{tabular}{|l|c|c|c|c|}
\hline \multirow{2}{*}{ Dental pathology type } & \multicolumn{2}{|c|}{$\begin{array}{c}\text { Decorative rabbits } \\
(\mathbf{n = 2 3 5 )}\end{array}$} & \multicolumn{2}{c|}{$\begin{array}{c}\text { Farm rabbits } \\
(\mathbf{n}=\mathbf{3 8 3})\end{array}$} \\
\cline { 2 - 5 } & young & old & young & old \\
\hline Overgrowth of incisors & $43.2 \%$ & $7.1 \%$ & $3.5 \%$ & $1.6 \%$ \\
\hline Overgrowth of cheek teeth & $16.9 \%$ & $80.6 \%$ & $2.7 \%$ & $9.7 \%$ \\
\hline $\begin{array}{l}\text { Uneven wear due to tooth } \\
\text { loss }\end{array}$ & $5.7 \%$ & $75.9 \%$ & $7.4 \%$ & $10.1 \%$ \\
\hline
\end{tabular}

Comparative statistics of the incidence of three types of dental pathologies in decorative and agricultural rabbits of different age groups are presented in Table 2.

Table 2. Comparison of the incidence of various dental pathologies in young and old individuals of decorative and farm rabbits.

\begin{tabular}{|c|c|c|}
\hline $\begin{array}{c}\text { Dental } \\
\text { pathology type }\end{array}$ & $\begin{array}{c}\text { Decorative rabbits } \\
(\mathbf{n}=\mathbf{2 3 5})\end{array}$ & $\begin{array}{c}\text { Farm rabbits } \\
(\mathbf{n}=\mathbf{3 8 3})\end{array}$ \\
\hline
\end{tabular}




\begin{tabular}{|l|c|c|c|c|}
\hline & young & old & young & old \\
\hline $\begin{array}{l}\text { Overgrowth of } \\
\text { incisors }\end{array}$ & $82.2 \%$ & $17.8 \%$ & $45.1 \%$ & $54.9 \%$ \\
\hline $\begin{array}{l}\text { Overgrowth of } \\
\text { cheek teeth }\end{array}$ & $28.9 \%$ & $71.1 \%$ & $19.8 \%$ & $80.2 \%$ \\
\hline $\begin{array}{l}\text { Uneven wear } \\
\text { due to tooth loss }\end{array}$ & $9.4 \%$ & $90.6 \%$ & $4.9 \%$ & $95.1 \%$ \\
\hline
\end{tabular}

Tables 1 and 2 show that dental pathologies occupy a significant proportion of all registered rabbit diseases in the Saratov Region. Most often, dental diseases appear in older animals, with the exception of the overgrowth of incisors, this pathology is often found in young rabbits. Table 1 shows that dental problems, among all other pathologies, are more common in decorative rabbits, however, this is due to the fact that the owners of farm rabbits with symptoms such as refusal to feed, exhaustion, swelling, reject the animals and slaughter them.

\section{Discussion}

Treatment for overgrowth of cheek teeth consists of removing the sharp ends of the teeth hooks and spines. Individual teeth are removed if their roots are infected and cause abscesses. Correction of cheek teeth is carried out most often for decorative rabbits for apartment maintenance. Less often, it is performed for high-yield agricultural rabbits, producers, if their pathology was not caused by a genetic predisposition, but was the result of injury or improper feeding. Simple filing of the incisors is carried out without sedation, using a secure fixation of the rabbit. If the incisors grow too quickly in the rabbit, then they are extracted.

When the diet of rabbits is lacking in fiber, teeth grow faster than they wear out, resulting in uneven tooth length, misalignment (malocclusion), abnormal curvature, sharp ends (spurs/spikes), loose molars, sharp tooth tips that damage the surrounding fabrics $[1$, 3].

The main way to prevent dental disease in a rabbit is to provide an appropriate diet. The main components of the diet shall be: a large amount of grass and good quality hay to provide a large amount of fiber and mineral particles for active wear of the molars. Fiber shall make up the bulk of their diet. They also require a small amount of dry food, which is recommended to be used in pellet form to prevent selective feeding that provides them with essential vitamins and minerals.

Traumatic injury is the second additional cause of dental disease in rabbits. Therefore, the correct organization of cells and the space in them can reduce the traumatism of animals [6-8].

Dental diseases in rabbits can also be hereditary in nature. Early detection and treatment of dental problems in rabbits requires regular dental examinations and evaluations [7-8].

\section{Conclusion}

Dental pathologies occupy a large share among all the noted diseases of rabbits in the Saratov Region. Most often, dental diseases occur in older animals, with the exception of the overgrowth of incisors, this pathology is often found in young rabbits. The main etiological factors in the occurrence of dental diseases in rabbits are improper feeding, dentoalveolar trauma and/or genetic predisposition.

The protocol for the treatment of dental pathologies is based on the timely correction of incisors and cheek teeth. In severe cases, the patient requires the stabilization before 
anesthesia with supportive therapy, including intravenous fluids, pain relief, and adequate nutritional support to support bowel motility. Rabbits with anorexia and dehydration are given supportive care such as intravenous fluids, pain relief, and supportive feeding prior to treatment. If antibiotics are needed to fight an infection

The complex of preventive measures consists in controlling the diet of rabbits, in which there shall be a large amount of grass and good quality hay in order to provide a sufficient amount of fiber and mineral particles for active wear of the molars. Also, the correct organization of cages and the space in them is required in order to reduce injuries to animals.

To exclude hereditary dental pathologies, an examination of sires before mating and the removal from reproduction of animals with genetically determined dental pathologies is required. For high-value sires with non-hereditary dental pathologies, tooth correction is economically feasible.

\section{References}

1. N.B. Nikulina (IPC «Prokrost"», Perm', 2019)

2. V. G. Kahikalo, O. V. Nazarchenko, A. A. Balandin (Lan', Sankt-Peterburg, 2021)

3. E.A. Alekseeva (KrasGAU, Krasnoyarsk, 2016)

4. S.V. Vorobievskaya, M.I. Stacenko, Ippologiya i veterinariya, 30, 4 (2018)

5. M.YU. Metlyakova, Nauka, innovacii i obrazovanie v sovremennom APK: sbornik mat. mezhd. nauch.-prakt. konf. (Izhevskoj GSKHA v 3-h tomah, Izhevsk, 2014)

6. N. N. SHumilina, YU. A. Kalugin, N. A. Balakirev (Lan', Sankt-Peterburg, 2016)

7. A. Meredith, B. Lord, BSAVA Manual of Rabbit Medicine (2016)

8. J. Chitty, BSAVA Manual of Rabbit Surgery, Dentistry and Imaging, Edition Frances Harcourt-Brown (2016)

9. E. Keeble, BSAVA Manual of Wildlife Casualties, Edition Elizabeth Mullineaux (2017)

10. N. V. Zelenevskij, M. V. SHCHipakin (Lan', Sankt-Peterburg, 2021)

11. N. V. Zelenevskij, M. V. SHCHipakin, K. N. Zelenevskij (Lan', Sankt-Peterburg, 2020)

12. A. V. ZHarov (Lan', Sankt-Peterburg, 2021)

13. C. A. Artiles, Journal of the American Veterinary Medical Association, 257, 3 (2020)

14. B. L. Oglesbee, Exotic Animal Laboratory Diagnosis (2020)

15. E.V. Baglikova, D.I. Ishchenko, A.I. Blednov, materialy Vserossijskoj (nacional'noj) nauchno-prakticheskoj konferencii, posvyashchennoj 100-letiyu so dnya rozhdeniya professora A.A. Sysoeva (Izhevsk, 2020)

16. I. A. Nikulin, S. P. Kovalev, V. I. Maksimov, YU. A. SHumilin (Lan', SanktPeterburg, 2020)

17. J. Hedley Antibiotic usage in rabbits and rodents, 40, 6 (2018) 